\title{
Estudio psicométrico de las escalas de depresión, ansiedad y funcionalidad familiar en estudiantes de la Universidad Industrial de Santander
}

\author{
Alimar Benitez Molina ${ }^{1}$ y María Claudia Caballero Badillo ${ }^{12}$. \\ ${ }^{1}$ Universidad Pontificia Bolivariana, seccional Bucaramanga, Colombia, ${ }^{2}$, Universidad Industrial de Santander, \\ División de Bienestar Universitario, Colombia.
}

Recibido, mayo 29/2015

Concepto de evaluación, mayo 2/2016

Aceptado, noviembre 24/2016
Referencia: Benítez Molina, A. \& Caballero Badillo, M.C. (2017). Estudio psicométrico de las escalas de depresión, ansiedad y funcionalidad familiar en estudiantes de la Universidad Industrial de Santander. Acta Colombiana de Psicología, 20(1), 221-231. DOI: 10.14718/ACP.2017.20.1.11

Resumen

Se estudiaron las propiedades psicométricas de los cuestionarios de depresión y ansiedad de Zung y el APGAR familiar, aplicados por la División de Bienestar Universitario (DBU) de la Universidad Industrial de Santander (UIS), como parte de la evaluación integral de los estudiantes admitidos para el diagnóstico temprano de trastornos que puedieran influir en su desempeño académico. Se tomó una muestra de 3614 estudiantes con edades entre 15 y 56 años que ingresaron en las cohortes de 2013 y 2014. Se verificaron las propiedades psicométricas de los instrumentos, y los resultados fueron comparados según sexo y edad. Se observaron niveles de confiabilidad de $.85, .842$ y .875 para las escalas de ansiedad, depresión y APGAR Familiar, respectivamente, aunque se requirió la modificación de la escala de depresión con la eliminación del ítem 6 . La correlación lineal de Spearman entre ansiedad y depresión fue de $\mathrm{r}=.76$, y de $\mathrm{r}=-.526$ entre el APGAR familiar y depresión, lo que indica una adecuada validez convergente y divergente. Al comparar los diagnósticos clínicos realizados en la consulta general de psicología y la consulta especializada de psiquiatría realizada por la Sección Servicios Integrales de Salud de la DBU con los puntajes de las pruebas, se observaron niveles de sensibilidad de 70, 67 y $67 \%$ en ansiedad, depresión y APGAR familiar, respectivamente. Finalmente, se encontró que en promedio las mujeres presentaron mayores puntajes de ansiedad y depresión en comparación con los hombres, y una funcionalidad familiar similar entre ambos sexos, sin diferencias significativas por edad.

Palabras clave: Psicometría, APGAR familiar, ansiedad y depresión de Zung, baremos.

\section{Psychometric Study of the Depression, Anxiety and family Dysfunction Scales in Students at Universidad Industrial de Santander}

\begin{abstract}
The aim of this research is to study the psychometric properties of Zung's and Apgar's Questionnaires for Depression and Anxiety, applied by the División de Bienestar Universitario de la UIS (DBU, for its Spanish acronym), a division in charge of students' welfare at UIS. The questionnaires were applied as part of a comprehensive assessment of admitted students, oriented to an early diagnosis of risk factors that may influence their academic performance. The sample consisted of a group of 3614 students admitted to the 2013 and 2014 cohorts, ages between 15 and 56 years. The psychometric properties of the instruments were verified and the results were compared by gender and age. Confidential levels of $0,85,0,842$ and 0,875 were observed for anxiety, depression and Family Apgar Scales, respectively. Only the item 6 of the depression test was eliminated since it did not show an adequate discrimination level. Spearman lineal correlation between anxiety and depression was $r=0,76$ and, $r=0,526$ between Family Apgar and depression. Those values indicate adequate convergent and divergent validity. Once psychometric validity was verified, results were used to establish a comparison with clinical diagnoses obtained from general and specialized psychiatric consultation carried out by DBU. Sensitivity levels of $70 \%$ for anxiety and depression and $67 \%$ for Family Apgar tests were found. Finally, higher anxiety and depression were found in the women's group compared to the men's, and a similar level of family dysfunction in both groups. No significant differences were observed between students' age groups.

Key words: Zung Anxiety and Depression Tests, Family Apgar terst, Psychometrics.
\end{abstract}

\footnotetext{
* Universidad Industrial de Santander (UIS), División de Bienestar Universitario. Carrera 27 Calle 9, Edificio de Bienestar Universitario, oficina 309-5, Bucaramanga. macaba@uis.edu.co

La presente investigación se desarrolló gracias a la OPS N²014003796 (UIS-Colombia) a cargo de la primera autora. Este trabajo ha sido presentado al equipo del Sistema de Excelencia Acémica de la UIS como parte del cierre de la contratación.
} 


\title{
Estudo psicométrico das escalas de depressáo, ansiedade e funcionalidade familiar em estudantes da Universidade Industrial de Santander
}

\begin{abstract}
Resumo
Estudaram-se as propriedades psicométricas dos questionários de depressão e ansiedade de Zung e o APGAR familiar, aplicados pela Divisão de Bem-estar Universitário (DBU) da Universidad Industrial de Santander (UIS), como parte da avaliação integral dos estudantes admitidos para o diagnóstico precoce de transtornos que pudessem influir em seu desempenho acadêmico. Tomou-se uma amostra de 3614 estudantes com idades entre 15 e 56 anos que ingressaram nas turmas de 2013 e 2014. Verificaram-se as propriedades psicométricas dos instrumentos, e os resultados foram comparados segundo sexo e idade. Observaram-se níveis de fiabilidade de $.85, .842$ e .875 para as escalas de ansiedade, depressão e APGAR familiar respectivamente, ainda que se tenha solicitado a modificação da escala de depressão com a eliminação do item 6 . A correlação linear de Spearman entre ansiedade e depressão foi de $r=.76$, e de $r=-.526$ entre o APGAR familiar e depressão, o que indica uma adequada validade convergente e divergente. Ao comparar os diagnósticos clínicos realizados na consulta geral de psicologia e na consulta especializada de psiquiatria realizada pela Seção Serviços Integrais de Saúde da DBU com as pontuações das provas, observaram-se níveis de sensibilidade de 70, 67 e 67\% em ansiedade, depressão e APGAR familiar, respectivamente. Finalmente, constatou-se que em média as mulheres apresentaram maiores pontuações de ansiedade e depressão em comparação com os homens, e uma funcionalidade familiar similar entre ambos os sexos, sem diferenças significativas por idade.

Palavras-chave: ansiedade e depressão de Zung, APGAR familiar, baremas, psicometria.
\end{abstract}

\section{INTRODUCCIÓN}

En su libro How College Affects Students, Pascarella y Terenzini (1991) mencionaron cuatro factores a tener en cuenta para el éxito o fracaso académico de los nuevos aspirantes a cursar estudios universitarios: el prestigio y calidad de la universidad seleccionada, la certeza sobre la carrera escogida, los recursos económicos disponibles para cursar los estudios, y la integración social que se alcance dentro del campus universitario. La Universidad Industrial de Santander (UIS) no interviene en los dos primeros aspectos, pero sí lo hace en los dos últimos con la División de Bienestar Universitario (DBU), la cual tiene como propósito principal promover la formación integral y el mejoramiento de la calidad de vida de los estudiantes admitidos para cursar estudios dentro de esta institución educativa. En este sentido, la DBU se encarga del diagnóstico de los estudiantes al momento de su ingreso para identificar rasgos de personalidad, habilidades, hábitos de consumo de sustancias psicoactivas, estado de salud física y vulnerabilidad económica a través de un enfoque biopsicosocial con el fin de fomentar en ellos el autocuidado y la cultura de la prevención, e intervenir oportunamente ante la presencia de factores que puedan condicionar al estudiante al fracaso escolar.

Investigaciones previas afirman que la depresión y la ansiedad pueden ser consideradas como factores que influyen negativamente en la integración del estudiante a la vida universitaria y, en consecuencia, en su desempeño académico (Jaude, 2002). Además, se ha encontrado que las profundas disfunciones familiares pueden propiciar conductas suicidas en los adolescentes (Larraguibel, González, Martínez, \& Valenzuela, 2000), lo que claramente perjudica su integración social y desempeño escolar.

Al respecto, otros autores, como Bermúdez et al. (2005), Paz y Aymat (2007) y Naranjo Quizhpi, Ñauta Uzhca y Ñauta Uzhca (2014), encontraron que las relaciones familiares deficientes y la falta de apoyo familiar dificultan el éxito académico de los estudiantes; esto teniendo en cuenta que el entorno universitario está generalmente asociado a nuevos compromisos, responsabilidades, situaciones estresantes y desplazamiento forzoso; aspectos que pueden limitar el acceso a una red de apoyo social y familiar, y que, sumados a circunstancias económicas precarias, pueden desencadenar síntomas depresivos y ansiosos en algunos individuos que afectan su desempeño escolar (Balanza, Morales, \& Guerrero, 2009).

La prueba de ingreso de la DBU mide rasgos de personalidad, dentro de los cuales se encuentran la ansiedad, la depresión, la funcionalidad familiar y el consumo de alcoholy sustancias psicoactivas, entre otras características individuales. Esta medición se hace con el propósito de identificar los factores de riesgo que puedan condicionar a los estudiantes al fracaso, para brindarles atención temprana, incrementar su permanencia en la universidad y mitigar los problemas relacionados con la deserción.

Los instrumentos psicométricos utilizados para determinar los niveles de ansiedad y depresión fueron diseñados originalmente en inglés por Zung $(1965,1971)$ y posteriormente traducidos y aplicados en numerosos estudios en población latinoamericana (Hernández, Macías, Callejas, Cerezo, \& Chauvet, 2008); y colombiana (Bermúdez, et al., 2005; 
Campo-Arias, Díaz-Martínez, Rueda-Jaimes, \& BarrosBermúdez, 2005; Cogollos, Días, \& Campos, 2006; De la Ossa, Martinez, Herazo, \& Campos, 2009; Lezama, 2012).

Por ejemplo, De La Ossa, et al. (2009), realizaron una investigación con 221 estudiantes con edad promedio de 20.5 años ( $\mathrm{DE}= \pm 2.6$ años) de las carreras de medicina y psicología en la ciudad de Cartagena, Colombia, para verificar las propiedades psicométricas de la escala de ansiedad de Zung, y encontraron un alfa de Cronbach $r=.77$ con tres factores que explicaron el $40.1 \%$ de la variabilidad total.

Para la escala de depresión de Zung, en un estudio de Cogollos, et al. (2006), fueron reportadas las propiedades psicométricas de la escala en 408 adolescentes entre 13 y 17 años de edad en la ciudad de Cartagena, en donde encontraron una consistencia interna de $r=.689$ y una estructura factorial de dos dimensiones que explicaron el $24.9 \%$ de la variabilidad total. Asimismo, Campos-Ríos et al. (2005) aplicaron el instrumento a un grupo de 110 universitarias con edad promedio de 22.1 años ( $\mathrm{DE}= \pm 3.4$ años) en la ciudad de Bucaramanga y encontraron una alfa de Cronbach de $r=.85$; además, los autores compararon los resultados con el diagnóstico clínico de depresión y reportaron una sensibilidad de $94.7 \%$, y una especificidad de $67 \%$, $\mathrm{VPP}=37.5 \%$ y VPN $=98.4 \%$.

Y con respecto al instrumento para medir la funcionalidad familiar empleado por Bienestar Universitario, conocido como APGAR familiar, este ha sido aplicado por varios investigadores en muestras colombianas (Forero Ariza, Avendaño Durán, Duarte Cubillos, \& Campo-Arias, 2006; Gómez Bustamante, Castillo Ávila, \& Cogollo, 2013), en donde se han encontrado valores de consistencia interna muy similares, con puntuaciones de alfa de Cronbrach alrededor de .78 .

Con respecto a este proceso diagnóstico desarrollado por la DBU de los estudiantes admitidos, surgen los siguientes interrogantes: primero, ¿los cuestionarios usados tienen propiedades psicométricas apropiadas para la población objetivo?, segundo, ¿las puntuaciones de ansiedad, depresión y funcionalidad familiar son similares entre grupos de sexo?, y, por último, ¿'los niveles de ansiedad, depresión y funcionalidad familiar en los estudiantes de nuevo ingreso son diferentes entre rangos de edades? Para dar respuesta a estas preguntas, y con el fin de optimizar las posibilidades de éxito en los estudiantes admitidos y garantizar que las mediciones sean acertadas y ajustadas a las características propias de la población objeto, se proponen los siguientes objetivos: a) estudiar las propiedades psicométricas de los cuestionarios de ansiedad de Zung (1971), depresión de Zung (1965) y funcionalidad familiar de Smilkstein (1978, en Suarez \& Alcalá, 2014); y b) comparar las puntuaciones de ansiedad, depresión y funcionalidad familiar entre sexos y rangos de edad de los estudiantes.

\section{MÉTODO}

\section{Tipo de investigación}

La presente investigación se llevó a cabo mediante un diseño no experimental de tipo instrumental (CarreteroDios \& Pérez, 2005), ya que no hubo manipulación sobre las variables evaluadas.

\section{Participantes}

La muestra estuvo conformada por 3614 estudiantes de la UIS. De estos, 1110 (30.7\%) fueron admitidos en el segundo período del 2013, 1459 (40.4 \%) eran del primer periodo del 2014, y 1045 (28.9\%) del segundo periodo del 2014. Del total de admitidos en los tres periodos, el $43.4 \%$ eran del sexo femenino y $56.6 \%$ de sexo masculino; y contaban con edades comprendidas entre los 15 y 56 años: $55.2 \%$ tenían 17 años o menos, $31 \%$ entre 18 y 19 años, y $13.9 \% 20$ años o más; el promedio de edad fue de 18.01 años $(\mathrm{DE}= \pm 2.34)$.

\section{Instrumentos}

APGAR Familiar. Es un cuestionario diseñado por el neurólogo Gabriel Smilkstein en 1978 que consta de cinco afirmaciones con escala de respuesta likert (nunca $=0$; siempre $=4)$. Este instrumento mide el grado de funcionalidad familiar (o disfuncionalidad) que el miembro de la familia, se supone, está en capacidad de percibir (Suarez \& Alcalá, 2014). En una validación realizada en una muestra colombiana de 91 estudiantes se encontró un alfa de Cronbach de $r=.793$, y una estructura unifactorial que explicó el $55.6 \%$ de la variabilidad total de la escala (Forero, Avendaño, Duarte, \& Campos, 2006).

Escala de Depresivos de Zung. Esta escala, creada por Zung en 1965, está basada en aspectos afectivos, fisiológicos y psicológicos que generalmente caracterizan la depresión, y está conformado por 20 reactivos con respuestas en formato tipo likert (nunca $=1$; siempre $=4$ ), en donde los ítems 2 , $5,6,11,12,14,16,17,18$ y 20 se encuentran redactados en afirmaciones negativas. En Colombia, Lezama (2012) realizó un estudio para determinar las propiedades psicométricas de la escala en una muestra de 4407 jóvenes entre 12 y 18 años, en donde encontró un alfa de Cronbach de $r=.548$, y una estructura factorial de cuatro dimensiones: 
síntomas afectivos, síntomas físicos, síntomas cognitivos y síntomas psicológicos, que explicaron el $13.55 \%, 11.61 \%$, $9.72 \%$ y $8.68 \%$, respectivamente, de la variabilidad total de la escala.

Escala de Ansiedad de Zung. Instrumento diseñado por Zung en 1971, originalmente en inglés y posteriormente traducido al español (De La Ossa, Martínez, Herazo \& Campos, 2009). Esta escala está estructurada en 20 afirmaciones con escala de respuesta tipo likert (nunca $=1$; siempre $=4$ ), donde las preguntas 5, 9, 13, 17 y 19 están redactadas en afirmaciones negativas. Un puntaje alto indica una alta disposición a la ansiedad, lo cual, según Hernández et al. (2008), se define como "una tendencia a presentar una sensación difusa y vaga de aprehensión o preocupación" (p. 20). Este instrumento fue validado en una muestra mexicana de 920 personas con edades entre los 14 y 60 años, donde se encontró un alfa de Cronbach de $r=.77 \mathrm{y}$ una estructura factorial de cuatro dimensiones que explicaron el $45.5 \%$ de la variabilidad total (Hernández et al., 2008).

\section{Análisis de datos}

Los datos fueron recolectados por la UIS con el software SIMSIS de la Sección de Servicios Integrales de Salud de Bienestar Universitario, mediante la aplicación de un cuestionario digital auto-administrado que formó parte del proceso del examen de salud en el momento del ingreso a la universidad. Los datos de los estudiantes de las cohortes seleccionadas fueron suministrados por la DBU para el desarrollo del presente estudio.

Para dar respuesta a los interrogantes de la investigación, se inició la verificación de las propiedades psicométricas de las escalas mediante el estudio de la consistencia interna de las escalas, utilizando el alfa de Cronbach, y, posteriormente, haciendo un análisis factorial exploratorio.

El poder de discriminación de los ítems que hacen parte de cada una de las escalas se calculó mediante el área bajo la curva Característica de Operaciones del Receptor (ROC, por sus siglas en inglés), usando el ajuste no paramétrico debido al gran volumen de datos, y considerando los ítems como adecuados cuando presentaban un área significativamente mayor a .5. Adicionalmente, se utilizó la prueba Chi-cuadrado de Pearson para tablas de contingencia para medir la independencia entre los diagnósticos clínicos y su incidencia por año.

Por otro lado, se utilizó el índice de correlación lineal de Spearman para estudiar la validez convergente y divergente entre las escalas y dimensiones consideradas; y se hizo uso de la prueba t de Student para la comparación de medias entre dos grupos independientes, así como del ANOVA unifactorial en el caso de más de dos grupos independientes, y comparaciones a posteriori de Diferencia Mínima Significativa (DMS). El procesamiento estadístico se realizó con la ayuda del IBM SPSS Statistics 22 y el Stata 12.0, y el nivel de significación máximo para el contraste de las hipótesis estadísticas fue de .05 .

\section{Consideraciones éticas}

La información suministrada por la DBU fue el resultado de la aplicación de los test en el Examen de Ingreso de los estudiantes admitidos a la UIS. Los datos suministrados por diferentes dependencias de la Universidad fueron recibidos en archivos de Excel sin datos personales, excepto por los códigos de los estudiantes para facilitar el cruce entre las matrices. La recolección de los datos no representó ningún riesgo para los estudiantes a nivel médico o psicológico, ni se realizaron procedimientos de tipo invasivo o de intervención clínica, según como corresponde en el artículo 11, investigación sin riesgo, de la Resolución 008430 de 1993 (Ministerio de Salud, Colombia), ya que fue contestada online desde un computador adjudicado en las salas de computo de la División de Servicios de Información. Los resultados de la investigación se presentaron ante el equipo del Sistema de Excelencia Académica (SEA) de la UIS.

\section{RESULTADOS}

A continuación se presentan los resultados de la investigación según el orden de su obtención. En principio se da cuenta del nivel de confiabilidad y validez de los instrumentos

Tabla 1

Confiabilidad de las pruebas de ansiedad y depresión de Zung y el APGAR familiar

\begin{tabular}{ccccc}
\hline Escalas & Ítems & Alfa de Cronbach & r elemento-total & $\mathrm{r}<.30$ \\
\hline Ansiedad de Zung & 20 & .85 & $.28-.586$ & 13,17 y 18. \\
Depresión de Zung & 20 & .822 & $.172-.595$ & $6^{*}, 7$ y 8. \\
APGAR familiar & 5 & .875 & $.630-.728$ & - \\
\hline
\end{tabular}

Nota. Primer cohorte de 2014; N = 1459; * La correlación elemento total fue de - .145. 
utilizados como parte del proceso diagnóstico de los estudiantes de nuevo ingreso que lleva a cabo la División de Bienestar Universitario en la Universidad Industrial de Santander. Posteriormente, se muestran las puntuaciones que caracterizan a los estudiantes en ansiedad, depresión y APGAR familiar, teniendo en cuenta los resultados de las comparaciones por edad y sexo.

\section{Análisis de confiabilidad de las escalas}

Como resultado del análisis realizado se obtuvo que, tal como se puede observar en la Tabla 1, las tres escalas descritas muestran una adecuada consistencia interna $(>.8)$, con correlaciones elemento-total que, como en el caso de la escala de APGAR, varían entre .63 y .72, indicando así una alta relación entre los ítems que conforman la escala.

Para la escala de ansiedad, la correlación elemento-total fue baja en los ítems 13,17 y $18(\mathrm{r}<.3)$, mientras que para la escala de depresión, el ítem 6 mostró una correlación negativa con el total de la escala, y las preguntas 7 y 8 mostraron correlaciones elemento total menor a .3.

Por otra parte, la estructura factorial de la escala de ansiedad mostró un Índice de Adecuación Muestral KMO = .919, con una prueba de esfericidad adecuada para continuar con el análisis factorial $\left(X_{(190)}^{2}=13068,14 ; p=.000\right)$; el porcentaje de varianza retenido para cuatro factores fue de $42.64 \%$. Aunque en el análisis factorial se muestran adecuadas cargas factoriales, el alfa de Cronbach entre las dimensiones fue de $.733, .731, .511$ y .265, lo cual indica que las dimensiones resultantes no presentan una adecuada consistencia interna. Por esta razón, se opta por mantener la estructura unifactorial originalmente planteada (sin el ítem 6), y con esto, al considerar la escala como unidimensional, la varianza que explica su único factor es de $27.25 \%$.

Con respecto a la escala de depresión de Zung, esta no evidencia una estructura factorial multidimensional bien definida para el grupo de jóvenes universitarios estudiados. $\mathrm{Y}$ al igual que en la escala de ansiedad, los factores se retienen por el criterio del autovalor mayor a 1, a cuatro dimensiones, pero las cargas factoriales entre las dimensiones son ambiguas, por lo que se opta por mantener solo un factor, el cual explica el $26.16 \%$ de la variabilidad total.

Y por último, el instrumento APGAR familiar mostró una estructura unidimensional con un porcentaje de varianza explicada de $67.01 \%$, con comunalidades entre $57.3 \%$ y $73.7 \%(\mathrm{KMO}=.879)$.

La discriminación de los ítems dentro de un instrumento de medición se evalúa considerando que si un individuo obtiene puntajes altos en la prueba, tiene también altas posibilidades de obtener puntajes altos en los ítems que la integran; y de no haber diferencias en el puntaje para el ítem respecto al total de la prueba, se afirma que el ítem no tiene poder de discriminación, por lo que debe ser eliminado. Para comparar los ítems respecto al total de la prueba, se usan grupos extremos o con puntajes altos (por encima del percentil 72) y puntajes bajos (inferior al percentil 28) tal como lo sugieren autores como Backhoff, Larrazolo y Rosa (2000), y se comparan los puntajes a través de la metodología de Curva ROC.

A continuación se presentan los valores del área bajo la curva ROC con su estimación por intervalos para la escala de ansiedad de Zung (véase Tabla 2).

Los ítems de la escala muestran un adecuado nivel de discriminación respecto a los puntajes altos y bajos de la escala, excepto para los ítems 10, 12 y 17, los cuales muestran áreas bajo la curva inferiores a .7, aunque significativamente distintas de.5. Como resultado no se sugiere la modificación de este cuestionario de ansiedad para su aplicación en la población colombiana antes descrita.

Como se observa en la Tabla 3, el ítem 6 de la escala de depresión de Zung no muestra discriminación entre los puntajes altos o bajos de la escala total, $\mathrm{y}$, adicionalmente, el coeficiente de correlación elemento-total para ese ítem fue negativo, por estas razones se opta por la eliminación de este ítem de la escala para aplicaciones en muestras colombianas. Finalmente, el cuestionario quedó constituido por 19 ítems y se obtuvo una confiabilidad alfa de Cronbach de .842 .

Los ítems del APGAR familiar muestran un excelente nivel de discriminación respecto al puntaje total, con áreas bajo la curva ROC significativamente mayores a .5 y un nivel de significación de .01 (véase Tabla 4).

\section{Análisis de validez de las escalas}

Para establecer la validez de constructo de los instrumentos de medición se realizó la validación convergente, divergente y de criterio de las escalas mediante el establecimiento de relaciones entre los puntajes obtenidos en instrumentos estudiados previamente respecto a la incidencia de trastornos y dificultades registradas por la DBU, como los motivos de consulta en los servicios médico asistenciales del estudiante ofrecidos por la Sección Servicios de Salud de la universidad, que podían ser la depresión, la ansiedad, la falta de apoyo familiar y problemas académicos.

Para la validez convergente y divergente se utilizó la correlación lineal de Spearman $(\mathrm{n}=1459)$ entre las diferentes variables psicosociales estudiadas. Y para la validación de constructo de las variables se consideraron como correlaciones altas, positivas o negativas a los puntajes de $|r|>$.4, resaltados en la tabla 5 . 
Tabla 2

Discriminación de los ítems de la escala de ansiedad de Zung

\begin{tabular}{|c|c|c|c|}
\hline \multirow{2}{*}{ Ítems de la escala ansiedad de Zung } & \multirow{2}{*}{ Área } & \multicolumn{2}{|c|}{ IC asintótico del $95 \%$} \\
\hline & & LI & LS \\
\hline ¿Se ha sentido últimamente más nervioso y ansioso? & $.859 * *$ & .834 & .884 \\
\hline ¿Se ha sentido temeroso sin razón? & $.811^{* *}$ & .781 & .841 \\
\hline ¿Se ha irritado fácilmente o ha sentido pánico? & $.836 * *$ & .808 & .864 \\
\hline ¿Ha sentido que se está derrumbando? & $.780 * *$ & .749 & .812 \\
\hline ¿Ha sentido que nada malo va a pasar/que todo va bien? & $.786 * *$ & .756 & .816 \\
\hline ¿Se ha sentido tembloroso? & $.769 * *$ & .737 & .801 \\
\hline ¿Le ha dolido el cuello, la espalda o la cabeza? & $.851 * *$ & .827 & .876 \\
\hline ¿Se ha sentido débil y se cansa fácilmente? & $.884 * *$ & .861 & .908 \\
\hline ¿Se ha sentido calmado y puede mantenerse quieto? & $.854 * *$ & .829 & .880 \\
\hline ¿Ha sentido palpitaciones, taquicardia, últimamente? & $.691 * *$ & .655 & .726 \\
\hline ¿Se ha sentido últimamente mareado? & $.772 * *$ & .740 & .804 \\
\hline ¿Se ha desmayado o ha sentido síntomas de desmayo? & $.636^{* *}$ & 600 & .673 \\
\hline ¿Ha podido respirar con facilidad? & $.700 * *$ & .665 & .736 \\
\hline Ha sentido hormigueo/falta de sensibilidad en los dedos & $.743 * *$ & .710 & .777 \\
\hline ¿Ha sentido náuseas y malestar en el estómago? & $.844 * *$ & .817 & .871 \\
\hline ¿Ha orinado con mayor frecuencia de lo normal? & $.744 * *$ & .711 & .778 \\
\hline ¿Ha sentido sus manos secas y calientes? & $.675 * *$ & .639 & .711 \\
\hline ¿Se ha ruborizado con frecuencia? & $.721 * *$ & .687 & .755 \\
\hline ¿Ha dormido bien y descansado toda la noche? & $.837 * *$ & .810 & .864 \\
\hline ¿Ha tenido pesadillas? & $.762 * *$ & .730 & .793 \\
\hline
\end{tabular}

Nota. $\mathrm{IC}=$ intervalo de confianza; $\mathrm{LI}=$ límite inferior; $\mathrm{LS}=$ límite superior; $* * \mathrm{p}<.01$.

Tabla 3

Discriminación de los ítems de la escala de depresión de Zung

\begin{tabular}{|c|c|c|c|}
\hline \multirow{2}{*}{ Ítems de depresión de Zung } & \multirow{2}{*}{ Área } & \multicolumn{2}{|c|}{ IC asintótico del 95\% } \\
\hline & & LI & LS \\
\hline ¿Se siente triste o decaído? & $.819^{* *}$ & .789 & .848 \\
\hline ¿Se siente mejor en las mañanas? & $.729 * *$ & .695 & .764 \\
\hline ¿Tiene ganas de llorar o tiene periodos de llanto? & $.786^{* *}$ & .755 & .817 \\
\hline ¿Duerme a ratos en la noche o se despierta muy temprano? & $.763 * *$ & .731 & .795 \\
\hline ¿Está comiendo como siempre ha comido? & $.800 * *$ & .770 & .830 \\
\hline ¿Experimenta deseos sexuales? & .473 & .434 & .511 \\
\hline ¿Nota que esta adelgazado? & $.689 * *$ & .654 & .724 \\
\hline ¿Tiene dificultades para hacer del cuerpo? & $.674 * *$ & .638 & .710 \\
\hline ¿Tiene palpitaciones & $.710 * *$ & .676 & .745 \\
\hline ¿Se siente cansado sin razón aparente? & $.820 * *$ & .792 & .849 \\
\hline ¿Tiene su mente tan despejada como siempre? & $.904 * *$ & .882 & .925 \\
\hline ¿Encuentra fácil hacer las cosas que hacía antes? & $.860 * *$ & .835 & .886 \\
\hline ¿Se siente inquieto y no puede mantenerse tranquilo? & $.803 * *$ & .773 & .833 \\
\hline ¿Tiene confianza en el futuro? & $.859 * *$ & .834 & .885 \\
\hline ¿Está más irritable que de costumbre? & $.796^{* *}$ & .765 & .826 \\
\hline ¿Encuentra fácil tomar decisiones? & $.829 * *$ & .802 & .856 \\
\hline ¿Se siente útil y necesario? & $.859 * *$ & .833 & .884 \\
\hline ¿Encuentra agradable vivir? & $.697 * *$ & .662 & .732 \\
\hline ¿Cree que los demás descansaran si usted muriera? & $.662 * *$ & .626 & .698 \\
\hline ¿Disfruta actualmente de sus actividades cotidianas? & $.877 * *$ & .853 & .901 \\
\hline
\end{tabular}

Nota. $\mathrm{IC}=$ intervalo de confianza; $\mathrm{LI}=$ límite inferior; $\mathrm{LS}=$ límite superior; ${ }^{* *} \mathrm{p}<.01$. 
Tabla 4

Discriminación de los ítems de la escala de APGAR familiar

\begin{tabular}{|c|c|c|c|}
\hline \multirow{2}{*}{ Ítems de la escala de Apgar Familiar } & \multirow{2}{*}{ Área } & \multicolumn{2}{|c|}{ IC asintótico del 95\% } \\
\hline & & LI & LS \\
\hline $\begin{array}{l}\text { 1. ¿Me siento satisfecho con la ayuda que recibo de mi familia cuando tengo algún } \\
\text { problema y/o necesidad? }\end{array}$ & $.954 * *$ & .938 & .970 \\
\hline $\begin{array}{l}\text { 2. ¿Me siento satisfecho con la forma en que mi familia habla de las cosas y } \\
\text { comparte los problemas conmigo? }\end{array}$ & $.974 * *$ & .964 & .985 \\
\hline $\begin{array}{l}\text { 3. Me siento satisfecho con la forma como mi familia acepta y apoya mis deseos } \\
\text { de emprender nuevas actividades? }\end{array}$ & $.906^{* *}$ & .884 & .929 \\
\hline $\begin{array}{l}\text { 4. ¿Me siento satisfecho con la forma como mi familia expresa afecto y responde } \\
\text { a mis emociones como rabia, tristeza o amor? }\end{array}$ & $.971 * *$ & .959 & .982 \\
\hline $\begin{array}{l}\text { 5. ¿Me siento satisfecho con la manera como compartimos en mi familia: el } \\
\text { tiempo para estar juntos, los espacios en la casa o el dinero? }\end{array}$ & $.962 * *$ & .949 & .976 \\
\hline
\end{tabular}

Nota. $\mathrm{IC}=$ intervalo de confianza; $\mathrm{LI}=$ límite inferior; $\mathrm{LS}=$ límite superior; $* * \mathrm{p}<.01$.

La ansiedad y la depresión son trastornos que afectan al individuo de manera negativa, razón por la cual se esperan correlaciones positivas entre los puntajes de ambas pruebas. Así, si una persona presenta puntajes elevados de ansiedad, esta también debería mostrar puntajes elevados de depresión, y si, por el contrario, un individuo muestra puntajes bajos de depresión, se espera que no evidencie síntomas o rasgos fuertes de ansiedad. En este estudio, la correlación entre estas variables fue de $.760(\mathrm{p}<.01)$, lo que confirma la validez convergente entre ambas pruebas.

Tabla 5

Matriz de correlaciones de Spearman entre las características psicosociales evaluadas

\begin{tabular}{|c|c|c|c|}
\hline & Ansiedad & Depresión & APGAR \\
\hline Depresión & $.760^{* *}$ & & \\
\hline APGAR familiar & $-.471^{* *}$ & $-.526^{* *}$ & \\
\hline
\end{tabular}

Nota. $* \mathrm{p}<.05 ; * * \mathrm{p}<.01$.

Por otro lado, se esperan correlaciones negativas entre la ansiedad y la depresión con respecto al apoyo familiar, esto es confirmado con coeficientes de correlación negativos e inferiores a $r=-.4$.

Adicionalmente, se compararon los puntajes totales en las diferentes escalas psicosociales utilizadas, con los motivos de consulta registrados por la DBU, especialmente en la consulta psiquiátrica y psicológica, en los que aparecen diagnósticos como "trastorno de ansiedad generalizada", "trastornos mentales y del comportamiento debidos al uso de cannabinoides: síndrome de dependencia", "trastorno de ansiedad no especificado", "trastorno mixto de ansiedad y depresión" y "episodio depresivo leve", entre otros, los cuales fueron clasificados y agrupados en las categorías de ansiedad (sí o no), niveles de depresión (generalizada, leve, moderada, severa y ausencia) y problemas de apoyo familiar (sí o no). En la tabla 6 se presentan los resultados de este apartado.

Los conflictos familiares se presentaron en aproximadamente $4 \%$ de los estudiantes admitidos en 2013, mientras que para los admitidos en 2014, la prevalencia fue de $1.7 \%$, lo que demuestra diferencias significativas entre estas dos proporciones.

Adicionalmente, de los admitidos en el 2013, $5.5 \%$ presentaron ansiedad, mientras que en 2014, $3.2 \%$ fueron diagnosticados clínicamente con este trastorno. Las proporciones mostraron diferencias significativas. Por otro lado, la depresión moderada se presentó en menor proporción en 2014, al igual que la depresión severa, pues $4.6 \%$ presentó este diagnóstico en 2013, mientras en 2014 disminuyó a $3.6 \%$; diferencias que resultaron ser significativas al nivel de .05 .

Cabe mencionar que $51.9 \%$ de los estudiantes que asistieron a la DBU con presencia de depresión también mostró presencia de ansiedad, lo cual es esperado debido a la correlación positiva y significativa mostrada en la tabla 5.

Para la validación de criterio, se compararon los puntajes totales de los instrumentos con la presencia de los diagnósticos asociados, de esta forma, los puntajes de la escala de depresión de Zung se compararon con el diagnóstico de depresión en estudiantes que acudieron a consulta, los puntajes en la escala de ansiedad de Zung con la presencia o ausencia de ansiedad de los estudiantes atendidos, y los puntajes en el APGAR familiar entre estudiantes diagnosticados con problemas familiares (véase Tabla 7).

El poder de discriminación de las escalas de ansiedad, depresión y APGAR familiar se evidenció con el área bajo la curva ROC, puntajes que resultaron significativamente mayores a .5. Adicionalmente, se observa que los niveles de sensibilidad y especificidad para los puntos de corte sugeridos son superiores a $50 \%$ en todos los casos. Los 
Tabla 6

Prevalencia de trastornos psicológicos registrados por Bienestar Universitario de la UIS en 2013 y 2014

\begin{tabular}{|c|c|c|c|c|c|c|c|}
\hline \multirow{3}{*}{\multicolumn{2}{|c|}{ Motivos de consulta }} & \multicolumn{4}{|c|}{ Año de admisión } & \multicolumn{2}{|c|}{ Chi-cuadrado de Pearson } \\
\hline & & \multicolumn{2}{|c|}{2013} & \multicolumn{2}{|c|}{2014} & \multirow{2}{*}{$X^{2}$} & \multirow{2}{*}{$\mathrm{p}$} \\
\hline & & $\mathrm{N}^{\circ}$ & $\%$ & $\mathrm{~N}^{\circ}$ & $\%$ & & \\
\hline \multicolumn{2}{|l|}{ Conflictos familiares } & $44^{\mathrm{a}}$ & $4.0 \%$ & $43^{\mathrm{b}}$ & $1.7 \%$ & 15.58 & $.000 * *$ \\
\hline \multirow[t]{2}{*}{ Ansiedad (sí) } & & $61^{\mathrm{a}}$ & $5.5 \%$ & $79^{b}$ & $3.2 \%$ & 10.69 & $.001 * *$ \\
\hline & Generalizada & $2^{\mathrm{a}}$ & $.2 \%$ & $2^{\mathrm{a}}$ & $0.1 \%$ & & \\
\hline \multirow{4}{*}{ Depresión } & Leve & $32^{\mathrm{a}}$ & $2.9 \%$ & $62^{\mathrm{a}}$ & $2.5 \%$ & & \\
\hline & Moderada & $13^{\mathrm{a}}$ & $1.2 \%$ & $13^{\mathrm{b}}$ & $0.5 \%$ & & \\
\hline & Severa & $4^{\mathrm{a}}$ & $.4 \%$ & $1^{\mathrm{b}}$ & $0.0 \%$ & & \\
\hline & $\mathrm{Si} / \mathrm{no}^{\mathrm{c}}$ & $51^{\mathrm{a}}$ & $4.6 \%$ & $78^{\mathrm{b}}$ & $3.6 \%$ & 4.68 & $.030 *$ \\
\hline \multicolumn{2}{|l|}{ Total } & 1110 & $100 \%$ & 2504 & $100 \%$ & & \\
\hline
\end{tabular}

Nota. ${ }^{1}$ Chi-cuadrado corregido por continuidad; ${ }^{\mathrm{a}} \mathrm{y}^{\mathrm{b}}$ indican proporciones iguales por columna según la prueba de comparaciones de proporciones por columna corregida por Bonferroni; ${ }^{c}$ presencia/ausencia de depresión; ${ }^{*} \mathrm{p}<.05 ; * * \mathrm{p}<.01$.

Tabla 7

Capacidad de discriminación de las escalas de ansiedad y depresión de Zung y el APGAR familiar en estudiantes de la UIS en los años 2013 y 2014

\begin{tabular}{|c|c|c|c|c|c|c|}
\hline \multirow{2}{*}{ Prueba } & \multirow{2}{*}{ Punto de corte } & \multirow{2}{*}{$\begin{array}{l}\text { Área bajo la } \\
\text { curva }\end{array}$} & \multicolumn{2}{|c|}{ IC del 95\% para el área } & \multirow{2}{*}{$\mathrm{S}_{\mathrm{e}}$} & \multirow{2}{*}{$\mathrm{S}_{\mathrm{p}}$} \\
\hline & & & LI & LS & & \\
\hline APGAR & 19 & $.672 * *$ & .616 & .728 & $67 \%$ & $57 \%$ \\
\hline Ansiedad & 32 & $.6697 * *$ & .623 & .716 & $70 \%$ & $54.30 \%$ \\
\hline Depresión & 30 & $.665^{* *}$ & .614 & .716 & $70.54 \%$ & $51.48 \%$ \\
\hline
\end{tabular}

Nota. $\mathrm{S}_{\mathrm{e}}=$ sensibilidad; $\mathrm{S}_{\mathrm{p}}=$ especificidad; $\mathrm{LI}=$ límite inferior; $\mathrm{LS}=$ límite superior; ${ }^{* *} \mathrm{p}<.01$.

resultados anteriores permiten concluir que las pruebas mencionadas miden características coherentes con su definición teórica dentro de la población estudiantil colombiana. La sensibilidad y especificidad observada es aceptable, pero cabe resaltar que no todos los estudiantes admitidos fueron diagnosticados clínicamente y solo se tiene dicho diagnóstico de los que solicitaron los servicios de la DBU.

Comparación de las puntuaciones de las escalas según sexo y edad

Para determinar si los puntajes de las pruebas en estudio muestran diferencias significativas entre ambos sexos, se utilizó la prueba t de Student para grupos independientes, partiendo del supuesto de que cada grupo comparado (mujeres y hombres) cuenta con tamaños de muestra muy grandes, lo cual permitiría asumir normalidad asintótica en el estadístico de prueba t de Student gracias a la aplicación del Teorema Central del Límite planteado por LindebergLévy (Mayorga, 2004).

En la tabla 8 se comparan los promedios de las pruebas psicológicas aplicadas por la DBU entre sexos, reflejando diferencias para ansiedad y depresión. En los casos donde se evidencian diferencias significativas,

Tabla 8

Comparaciones promedio de las variables psicosociales según el sexo

\begin{tabular}{lccccc}
\hline & \multicolumn{3}{c}{ Medias } & \multicolumn{2}{c}{ Prueba T para la igualdad de medias } \\
\cline { 2 - 6 } & $\begin{array}{c}\text { Mujeres } \\
(\mathrm{n}=1569)\end{array}$ & $\begin{array}{c}\text { Hombres } \\
(\mathrm{n}=2045)\end{array}$ & $\mathrm{t}$ & $\mathrm{gl}$ & Sig. (bilateral) \\
\hline Ansiedad $^{2}$ & 33.06 & 30.95 & 9.87 & 3110.16 & $.000 * *$ \\
Depresión $^{2}$ & 31.50 & 29.57 & 8.343 & 3088.59 & $.000 * *$ \\
APGAR familiar $^{2}$ & 19.43 & 19.52 & -.593 & 3253.3 & .554 \\
\hline $\mathrm{n}$ & 1568 & 2046 & & & \\
\hline
\end{tabular}

Nota. ${ }^{*} \mathrm{p}<0.01 ;{ }^{1}$ se asumen varianzas iguales; ${ }^{2}$ se asumen varianzas diferentes. 
los puntajes promedio son mayores en las mujeres, lo cual se afirma con un nivel de confianza del $99 \%$.

En la Tabla 9 se muestran las comparaciones de las variables psicológicas ansiedad, depresión y APGAR familiar entre estudiantes de 17 años o menos, entre $18 \mathrm{y}$ 19 años, y 20 o más años, para esto se utilizó el ANOVA de un factor (grupos etarios), considerando a mujeres y hombres por separado. Nuevamente, la aplicación de la técnica estadística es apropiada gracias a los tamaños de muestra, dado que garantizan que el estadístico de prueba ( $\mathrm{F}$ de Snedecor) siga una distribución asintóticamente normal (Mayorga, 2004). No se observaron diferencias promedio estadísticamente significativas entre los grupos etarios en mujeres ni en hombres, en ninguna de las escalas; esto se afirma con un nivel de significación .05.

Al estudiar la confiabilidad de las escalas de ansiedad y depresión entre grupos de mujeres y hombres, se encontraron resultados casi idénticos en la escala de ansiedad con .848 en cada grupo de sexo; en cambio, la consistencia interna de la escala de depresión para las mujeres fue de .854, mientras que en hombres fue de .83. En todos los grupos anteriores los índices de alfa de Cronbach indicaron una muy buena consistencia interna.

\section{DISCUSIÓN}

En la presente investigación se observó una consistencia interna de $r=.85$ con una estructura unidimensional para la escala de ansiedad, resultado comparable con el hallado por De La Ossa et al. (2009), quienes encontraron una confiabilidad de $r=.77$ para esta escala con 221 estudiantes de medicina y psicología, sin embargo, ellos reportaron 3 factores que explicaban el $40.1 \%$ de la variabilidad total.

En este estudio, la consistencia interna de la escala de depresión fue de $r=.822$ con la eliminación del ítem 6 por no presentar adecuados niveles de correlación elemento-total ni discriminación; la escala quedó constituida finalmente por
19 ítems en una estructura unidimensional, esta estructura es muy similar a la encontrada por otros autores como CampoArias, Díaz-Martínez, Rueda-Jaimes y Barros-Bermúdez (2005) y Cogollo et al. (2006), con niveles bajos de correlación elemento total en el ítem 6 , sin la revisión de la discriminación de los ítems de la escala. En estos estudios con muestras colombianas se encontraron coeficientes de alfa de Cronbach para la escala de depresión de $r=.85$ y $r=.689$, respectivamente, sin la eliminación de ítems.

La funcionalidad familiar se midió con el APGAR, y se encontró una consistencia de $r=.875$, con una estructura unifactorial, donde los cinco ítems de la escala reportan altos niveles de correlación elemento-total y de discriminación, similar a lo encontrado por Forero et al. (2006) y por Gómez, Castillo y Cogollo (2013).

En general, se observó que las escalas de ansiedad, depresión y APGAR familiar funcionaron adecuadamente en los estudiantes de la UIS, excepto por la variación de la escala de depresión, que finalmente estuvo conformada por 19 ítems. La correlación elemento-total y los niveles de discriminación de los ítems fueron adecuados en las tres escalas, y las correlaciones entre las mismas mostraron una dirección adecuada; esto confirma su validez convergente $\mathrm{y}$ divergente.

Con respecto a las comparaciones según sexo y edad se encontró que los niveles de ansiedad fueron significativamente mayores en mujeres, y no se observaron variaciones promedio significativas entre los rangos de edades para cada sexo.

$\mathrm{Al}$ revisar la consistencia interna de las escalas de ansiedad y depresión entre los grupos de hombres y mujeres, no se observaron diferencias importantes, lo que permite concluir que las escalas pueden ser usadas de forma confiable en hombres y mujeres.

La verificación de las propiedades psicométricas de los instrumentos usados en el proceso diagnóstico de los estudiantes admitidos en la UIS permite conocer los puntajes que son normales o rigen en esta población; esto facilitará

Tabla 9

Comparación de los puntajes de ansiedad, depresión y APGAR Familiar según grupos etarios y sexo

\begin{tabular}{|c|c|c|c|c|c|c|c|c|c|c|}
\hline & \multicolumn{6}{|c|}{ Edad } & \multirow{2}{*}{\multicolumn{2}{|c|}{ ANOVA }} \\
\hline & & & \multicolumn{2}{|c|}{$<=17(\mathrm{n}=1994)$} & \multicolumn{2}{|c|}{$18-19(n=1120)$} & \multicolumn{2}{|c|}{$>=20(n=502)$} & & \\
\hline & & & Media & $\pm \mathrm{DE}$ & Media & $\pm \mathrm{DE}$ & Media & $\pm \mathrm{DE}$ & $\mathrm{F}$ & $\mathrm{p}$ \\
\hline \multirow{6}{*}{ Sexo } & \multirow{3}{*}{ Mujeres } & APGAR familiar & 19.20 & 4.73 & 19.73 & 4.54 & 19.75 & 4.48 & 2.52 & .080 \\
\hline & & Ansiedad & 33.27 & 6.62 & 32.86 & 7.01 & 32.54 & 6.88 & 1.16 & .315 \\
\hline & & Depresión & 31.60 & 7.21 & 31.65 & 7.48 & 30.53 & 7.43 & 1.67 & .188 \\
\hline & \multirow{3}{*}{ Hombres } & APGAR familiar & 19.47 & 4.20 & 19.68 & 4.32 & 19.35 & 4.88 & .78 & .458 \\
\hline & & Ansiedad & 31.11 & 5.54 & 30.86 & 6.18 & 30.57 & 6.41 & 1.17 & .311 \\
\hline & & Depresión & 29.73 & 6.00 & 29.43 & 6.36 & 29.32 & 7.12 & .78 & .459 \\
\hline
\end{tabular}


en futuras cohortes el diagnóstico de debilidades y el estudio de factores de riesgo asociados al rendimiento académico en estudiantes, así como también fomentará la continuación en el trabajo de validación de los demás instrumentos de medición usados en dicho proceso.

En este sentido, se sugiere continuar con la validación de los instrumentos de medición utilizados en el examen de ingreso de la UIS, y, posteriormente, realizar el estudio de los factores de riesgo asociados a los problemas de deserción y bajo rendimiento académico de los estudiantes con el fin de proponer correctivos tempranos que promuevan su permanencia en la universidad y el éxito en las carreras cursadas.

\section{REFERENCIAS}

Backhoff, E., Larrazolo, N., \& Rosa, M. (2000). Nivel de dificultad y poder de driscriminación del Examen de Habilidades y Conocimientos Básicos (EXHCOBA). Revista Electrónica de Investigación Educativa, 2(1), 11-29. Recuperado de http://redie.uabc.mx/redie/article/viewFile/15/26

Balanza, S., Morales, I., \& Guerrero, J. (2009). Prevalencia de Ansiedad y Depresión en una población de estudiantes universitarios: factores académicos y sociofamiliares acosiados. Clínica y Salud, 177-187. Recuperado de http://www. redalyc.org/pdf/1806/180613879006.pdf

Bermúdez, S., Duran, M., Escobar, A., Morales, A., Monroy, S., Ramírez, A., \& Castrillón, J. J. (2005). Factores que afectan el rendimiento académico y estrés en los estudiantes de la Facultad de Medicina de la Universidad de Manizales durante el periodo académico de enero-junio del 2005. Universidad de Manizales, Facultad de medicina, Centro de investigaciones. Recuperado de http://ridum.umanizales.edu.co:8080/jspui/bitstream/6789/1464/1/articulo $\% 20$ fr\%20para\%20rendimiento\%20academico.pdf

Campo-Arias, A., Díaz-Martínez, L. A., Rueda-Jaimes, G. E., \& Barros-Bermúdez, J. A. (2005). Validación de la escala de Zung para depresión en universitarias de Bucaramanga, Colombia. Revista Colombiana de Psiquiatría, 34(1), 54-62. Recuperado de http://www. scielo.org.co/scielo.php?script=sci_arttext\&pid=S003474502005000100004\&lng=en\&tlng=es.

Carretero-Dios, H., \& Pérez, C. (2005). Normas para el desarrollo y revisión de estudios instrumentales. International Journal of Clinical and Health Pychology, 5(3), 521-551. Recuperado el 15 de 05 de 2016, de http://www.aepc.es/ijchp/articulos_pdf/ijchp-158.pdf

Cogollos, Z., Días, C. E., \& Campos, A. (2006). Exploración de la validez de constructo de la escala de Zung para depresión en adolescentes escolarizados. Colombia Médica, 32(2), 102-106.

De la Ossa, S., Martinez, Y., Herazo, E., \& Campos, A. (2009). Study of internal consistency and factor structure of three versions of the Zung's rating instrument for anxiety disorders. Colombia Médica, 40(1), 71-77. Recuperado el 15 de marzo de 2015, de http://www. scielo.org.co/scielo.php?script=sci_arttext\&pid=S165795342009000100006\&lng=en\&tlng=en

Forero, L. M., Avendaño, M. C., Duarte, Z. J., \& Campos, A. (2006). Consistencia interna y análisis de factores de la escala APGAR para evaluar el funcionamiento familiar en estudiantes de básica secundaria. Revista Colombiana de Psiquiatría, 35(1), 23-29. Recuperado el 8 de Marzo de 2015, de http://www.scielo.org.co/scielo.php?script=sci_ arttext\&pid=S0034-74502006000100003\&lng=en\&tlng $=$ es.

Gómez, E. M., Castillo, I., \& Cogollo, Z. (2013). Predictores de disfunción familiar en adolescentes escolarizados. Revista Colombiana de Psiquiatría, 42(1), 78-80. Recuperado el 15 de marzo de 2015, de http://www. scielo.org.co/scielo.php?script=sci_arttext\&pid=S003474502013000100007\&lng=es\&tlng=es.

Hernández, M. R., Macías, D., Callejas, N., Cerezo, S., \& Chauvet, C. F. (2008). Propiedades psicométricas del inventario Zung del estado de ansiedad con mexicanos. Psychologia, 2(2), 19-46.

Jaude J, G. (2002). Factores psicológicos que predisponen al bajo rendimiento, al fracaso y a la deserción escolar. Estud. Pedagóg, 193-204. Recuperado de http://mingaonline.uach.cl/scielo.php?script=sci_arttext\&pid=S071807052002000100012\&lng=es\&nrm=iso

Larraguibel Q., M., González M., P., Martínez N., V., \& Valenzuela G., R. (2000). Factores de riesgo de la conducta suicida en niños y adolescentes. Revista Chilena de Pediatría, 183-191. Recuperado de http://www. scielo.cl/scielo.php? script $=$ sci_arttext\&pid $=\mathrm{S} 0370$ $41062000000300002 \& \operatorname{lng}=$ es\&tlng=es. $\quad 10.4067 / \mathrm{S} 0370-$ 41062000000300002

Lezama, S. R. (2012). Propiedades Psicométricas de la escala de Zung para síntomas depresivos en población Adolescente escolarizada colombiana. Phychol. Av. Discip., 6(1), 91101. Recuperado de http://www.scielo.org.co/pdf/psych/ v6n1/v6n1a08

Mayorga, J. H. (2004). Inferencia Estadística. Bogotá: UNIBIBLIO.

Ministerio de Salud de la Republica de Colombia, Resolución No. 008430 de 1993 (4 de marzo de 1993). Recuperado de https://www.invima.gov.co/images/pdf/medicamentos/resoluciones/etica_res_8430_1993.pdf

Naranjo Quizhpi, M. D., Ñauta Uzhca, L. E., \& Ñauta Uzhca, M. E. (2014). Prevalencias y consecuencias de la disfuncionalidad familiar en el rendimiento académico de los estudiantes de séptimo a décimo año de la Unidad Educativa Estados Unidos, Cuenca 2014. Tesis de pregrado, Universidad de Cuenca. Recuperado de http://dspace.ucuenca.edu. ec/handle/123456789/20550

Pascarella, E. T., \& Terenzini, P. T. (1991). How College Affects Students. San Francisco: Jossey-Bass. 
Paz, S., \& Aymat, A. C. (2007). Problemas en el desempeño escolar y su relación con el funcionalismo familiar en el alumno de EGB 1. Revista de la Facultad de Medicina, 2732. Recuperado de http://www.fm.unt.edu.ar/Servicios/publicaciones/revistafacultad/vol_8_n_1_2007/cap5.pdf

Suarez, M. A., \& Alcalá, M. (2014). APGAR Familiar: una herramienta para detectar disfunción familiar. Rev. Méd. La Paz, 20(1), 53-57. Recuperado de http://www.scielo.org.bo/ scielo.php?pid=S1726-89582014000100010\&script $=$ sci_ arttext
Universidad Industrial de Santander. (25 de 07 de 2016). Universidad Insdustrial de Santander. Recuperado de https:// www.uis.edu.co/webUIS/es/administracion/bienestarUniversitario/portafolioServicios.html

Zung W. W. (1965). A self-rating depression scale. Arch Gen Psychiatry, 12, 63-70.

Zung, W. W. (1971). A rating instrument for anxiety disorders. Psichosomatics, 12(6), 371-379. 